\title{
DESCRIPCIÓN DE UNA NUEVA ESPECIE DEL GÉNERO PRIONYCHUS SOLIER, 1835 DE MARRUECOS Y REDESCRIPCIÓN DE PRIONYCHUS ANTRHACINUS (MULSANT, 1856) Y PRIONYCHUS LUGENS (KÜSTER, 1850) (COLEOPTERA, TENEBRIONIDAE, ALLECULINAE)
}

\author{
J. M. Blanco Villero' \& J. A. Sáez Bolaño²
}

\section{RESUMEN}

J. M. Blanco Villero \& J. A. Sáez Bolaño. 2011. Descripción de una nueva especie del género Prionychus Solier, 1835 de Marruecos y redescripción de Prionychus anthracinus (Mulsant, 1856) y Prionychus lugens (Küster, 1850) (Coleoptera, Tenebrionidae, Alleculinae). Grael/sia, 67(2): 175-185.

En este trabajo se describe una nueva especie de Prionychus del norte de Marruecos; en concreto de la región de Nador, en las cercanías de Melilla. Prionychus alfonsoblancoi sp. nov. pertenece al grupo de especies que presentan el prosterno, por delante de las procoxas, en declive plano o algo convexo, élitros casi sin estrías, borde anterior del pronoto no rebordeado y coloración castaña. Estos caracteres hacen que en el ámbito iberomagrebí, $P$. alfonsoblancoi se aproxime únicamente a Prionychus lugens, especie de la que puede separarse claramente por la longitud de las antenas, el punteado del pronoto y la estructura del edeago. $P$. alfonsoblancoi representa la cuarta especie presente en Marruecos y, por extensión, en el norte de África. Dados los índices biométricos que actualmente se utilizan para describir las especies de esta subfamilia, se ha optado por redescribir Prionychus anthracinus y Prionychus lugens según los criterios actuales. Además se ha elaborado una clave dicotómica de las especies del género que habitan la península ibérica y el norte de África.

Palabras clave: Taxonomía; Especie Nueva; Marruecos; Prionychus; Alleculinae; Tenebrionidae; Coleoptera.

\section{ABSTRACT}

J. M. Blanco Villero \& J. A. Sáez Bolaño. 2011. A new species of Prionychus Solier, 1835 from Morocco and redescription of Prionychus anthracinus (Mulsant, 1856) and Prionychus lugens (Küster, 1850) (Coleoptera, Tenebrionidae, Alleculinae). Graellsia, 67(2): 175-185 (in Spanish).

In this work we describe a new species of Prionychus from northern Morocco, specifically in the region of Nador, near Melilla. Prionychus alfonsoblancoi sp. nov. belongs to those species in which the prosternum, ahead of procoxa, angles downards in a flat or slightly convex slope, the elytra have almost no striae, the anterior margin of pronotum has no border and they show a chestnut colouration. Within the lberian/Maghreb area, these characters make $P$. alfonsoblancoi resemble only $P$. lugens, a species from which it can be clearly separated by the length of the antennae, the pronotal punctuation and the structure of the aedeagus. $P$. 


\begin{abstract}
alfonsoblancoi is the fourth species present in Morocco and, by extension, in North Africa. Given the biometrics indices currently used to describe the species of this subfamily, it has been decided to re-describe Prionychus anthracinus and Prionychus lugens according to up-to-date standards. A dichotomous key to the species of the genus that inhabit the lberian peninsula and northern África has also been developed.
\end{abstract}

Key words: Taxonomy; New species; Morocco; Prionychus; Alleculinae; Tenebrionidae; Coleoptera.

\section{Introducción}

El género Prionychus Solier, 1835, de distribución paleártica, está representado únicamente por 10 especies válidas: Prionychus ater (Fabricius, 1775); Prionychus melanarius (Germar, 1813), ambas de distribución europea; Prionychus fairmairii (Reiche, 1860), especie conocida de Francia, Italia, España y Marruecos; Prionychus lugens (Küster, 1850), citada de Córcega, Sicilia, Marruecos y Argelia; Prionychus anthracinus (Mulsant, 1856), que habita Argelia, Túnez y Marruecos; Prionychus asiaticus (Fairmaire, 1892), conocida únicamente de Albania y Turquía; Prionychus delagrangei (Fairmaire, 1892), de Turquía; Prionychus cisteloides Seidlitz, 1896, distribuida por oriente próximo; Prionychus nitidissimus Pic, 1905, especie conocida únicamente de Anatolia central y Prionychus striatipennis Pic, 1909, citada de Bulgaria y Rumanía (Novák \& Pettersson, 2008; Sáez et al., 2010).

Por tanto, en el norte de África sólo se conocen con certeza las siguientes especies: P. lugens, $P$. anthracinus y $P$. fairmairii.

Durante el transcurso de un estudio previo (Sáez et al., 2010) hemos tenido la oportunidad de estudiar una cuarta especie norteafricana depositada en el Museo Nacional de Ciencias Naturales de Madrid y no determinada, cuya morfología no encajaba con ninguna de ellas y que describimos en el presente artículo.

Las únicas descripciones conocidas de dos de las especies presentes en el norte de África -P. lugens y $P$. anthracinus - son las originales de Küster (1850) y Mulsant (1856) respectivamente. Dichas descripciones no tienen en cuenta obviamente los criterios biométricos que se utilizan en la actualidad para describir las especies de esta subfamilia, así como tampoco el estudio de las genitalias masculinas. Por otra parte la descripción de Prionychus anthracinus es muy breve y omite muchos detalles morfológicos. Dado que estos datos son de importancia discriminatoria en la taxonomía interna del género y aprovechando el resto del material del mismo procedente del Museo Nacional de Ciencias Naturales de Madrid ya estudiado y publicado (Sáez et al., 2010), hemos optado por redescribir ambas especies, lo cual no invalida sino que amplía las descripciones originales.

\section{Material y Métodos}

El material estudiado de todas las especies se relaciona en el epígrafe de cada una de ellas y se conserva en el Museo Nacional de Ciencias Naturales de Madrid (MNCN).

Asimismo se ha estudiado numeroso material de comparación de las otras especies presentes en Europa occidental, tales como $P$. ater, $P$. fairmairii y P. melanarius, cuyo detalle puede consultarse en Sáez et al. (2010).

El estudio morfológico se efectuó sobre los ejemplares en seco mediante la observación con estereomicroscopio. Para la extracción de las correspondientes genitalias fue necesaria la rehidratación de los ejemplares, siendo montadas en las mismas cartulinas con resina D.M.H.F. Las mediciones fueron efectuadas mediante un ocular micrométrico acoplado al estereomicroscopio. Entre las mediciones efectuadas destacan por su importancia para la caracterización de las especies de Alleculinae, el denominado "índice ocular" y el "índice pronotal". El "índice ocular" se define como el cociente entre la mínima distancia dorsal entre los ojos y la anchura máxima dorsal de un extremo del ojo al otro, multiplicado por 100 (Campbell \& Marshall, 1965). El "índice pronotal" se define como la razón entre la longitud máxima del pronoto a lo largo de la línea sagital media, multiplicada por 100, y la anchura máxima del pronoto a nivel de los ángulos posteriores (Campbell, 1964). Estos índices sumados a la longitud de las antenas y las dimensiones relativas entre los distintos antenómeros y la morfología del edeago consti- 
tuyen, además de otros más tradicionales, datos hoy fundamentales para la descripción de nuevas especies de la subfamilia (Campbell, 1971; Novák, 2006, 2007a, 2007b, 2008a y 2008b).

Las microfotografías han sido tomadas con una cámara digital acoplada al estereomicroscopio. Los dibujos son originales de uno de los autores (J. Sáez).

\section{Resultados}

\section{Prionychus alfonsoblancoi sp. nov.}

HOLOTYPUS: Un macho depositado en el Museo Nacional de Ciencias Naturales de Madrid ( $\mathrm{n}^{\mathrm{o}}$ catálogo 30810). Porta dos etiquetas; la primera original, blanca, manuscrita, aparece rotulada: "Muley Bagdad / Beni Sicar / 21-II-1924- Candel" (Fig. 1). La segunda, impresa por el Museo: " $M N C N E$ Ent / $N^{o} C a t$. 30810". Se añaden dos; una tercera, por los autores: "Prionychus alfonsoblancoi / J. Blanco \& J. Sáez 2011 / HOLOTYPUS y una cuarta por el Museo: MNCN Cat. Tipos No 2133.

DESCRIPCIÓN DEL HOLOTYPUS (Fig. 2): Morfología general oval, de coloración castaño oscuro, con las patas, antenas y palpos ligeramente más claros. Talla: longitud total desde el borde del clípeo al ápex elitral: 7,6 mm; anchura máxima en la unión del tercio medio con el tercio posterior de los élitros: 3,4 $\mathrm{mm}$ (L/A: 2,23).

Cabeza: Longitud (visible): $1,15 \mathrm{~mm}$; anchura máxima (a nivel de los ojos): 1,35 mm. De la misma coloración general que el cuerpo, algo más clara en el área del clípeo. Clípeo deprimido con respecto a la frente, lo cual dibuja un fuerte declive clípeofrontal. Ojos salientes con una notable distancia interocular: $0,7 \mathrm{~mm}$, lo que ofrece un índice ocular de 51,8. Punteado de la cabeza fuerte y densa, menor en el clípeo; los puntos casi con el doble de diámetro que los del pronoto y de mayor diámetro que la distancia que los separa. Tegumento visible entre los puntos muy brillantes.

Antenas: Relativamente largas con una longitud total de 4,23 $\mathrm{mm}$, alcanzando la unión del tercio basal con el tercio medial del élitro (LT/LA: 1,79). Antenómeros fuertemente punteados y provistos de abundantes sedas. Sus longitudes desde la base al ápex (entre paréntesis relación L/A) son las siguientes: $0,35(2,33) ; 0,2(1,40) ; 0,37(2,74) ; 0,39$ $(2,22) ; 0,35(1,87) ; 0,40(2,22) ; 0,41(2,7) ; 0,43$ $(2,68) ; 0,42(2,43) ; 0,4(2,66) ; 0,50(3,17)$.

Palpos maxilares: Del mismo color que las antenas, patas y clípeo, con setas relativamente densas,

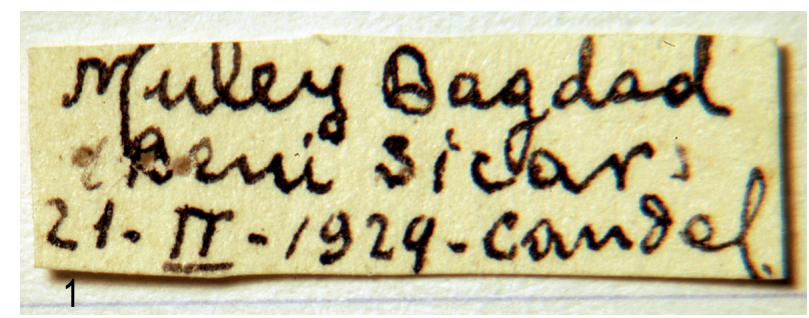

Fig. 1.- Prionychus alfonsoblancoi n. sp.: Etiqueta original del Holotypus.

Fig. 1.- Prionychus alfonsoblancoi $\mathrm{n}$. sp: Original label of the holotype.

especialmente en el último artejo donde, además, aparecen paralelas y tumbadas contra el tegumento. De ellos, el más corto es el penúltimo, el cual tiene una morfología triangular; el más largo el último o apical, también con morfología triangular. Las longitudes de los artejos $2^{\circ}$ a $4^{\circ}$ (entre paréntesis relación L/A) son las siguientes: 0,2 (1,77); 0,15 $(1,33)$; $0,35(1,86)$.

Pronoto: Coloración uniforme castaño oscuro como el resto del cuerpo. Transverso, algo menos del doble de ancho que de largo (longitud: 1,6 mm, anchura: $2,8 \mathrm{~mm}$, índice pronotal: 57,14$)$. Los bordes laterales se continúan insensiblemente con el borde anterior, de tal forma que prácticamente no existen ángulos anteriores, formándose casi un semicírculo rectificado por delante. Ángulos posteriores bien marcados pero romos y en ángulo ligeramente obtuso. Borde posterior y bordes laterales rebordeados, interrumpiéndose en el tercio medio del borde anterior. Visto lateralmente la convexidad pronotal es moderada, tanto en sentido anteroposterior como lateral. Punteado moderadamente denso y uniforme, de bordes levantados en áreas paramediales del disco a modo de cráteres. En el disco su densidad es inferior, de tal forma que el diámetro de los puntos es menor que la distancia que los separa; mientras que hacia los lados y ángulos posteriores, los puntos son más fuertes y densos, siendo su diámetro igual e incluso mayor que la distancia que los separa (Fig. 3). El tegumento visible entre los puntos muestra una neta microescultura que disminuye su brillo, ofreciendo un aspecto satinado. Sedas pronotales relativamente largas, de color castaño oscuro y tumbadas hacia atrás, más largas hacia los lados que en el disco. El pronoto muestra sendas leves depresiones en la región basal paramedial. 


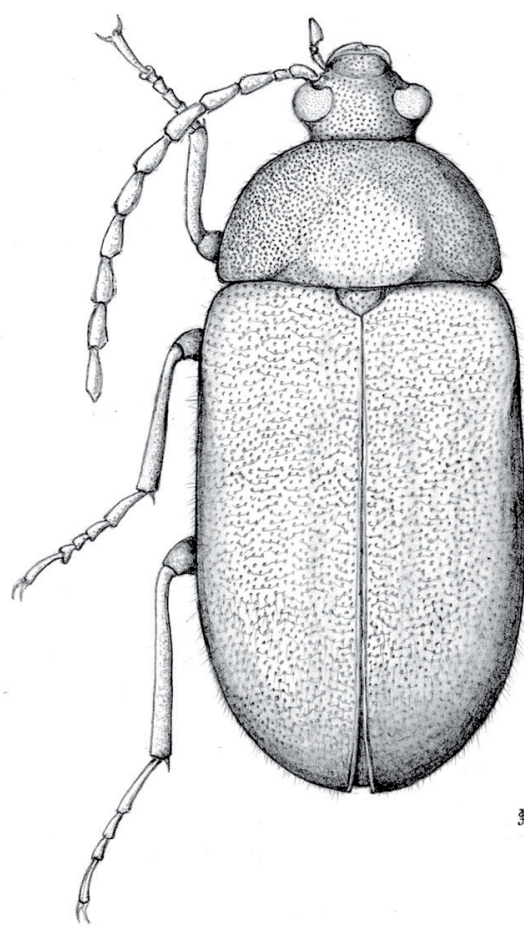

2

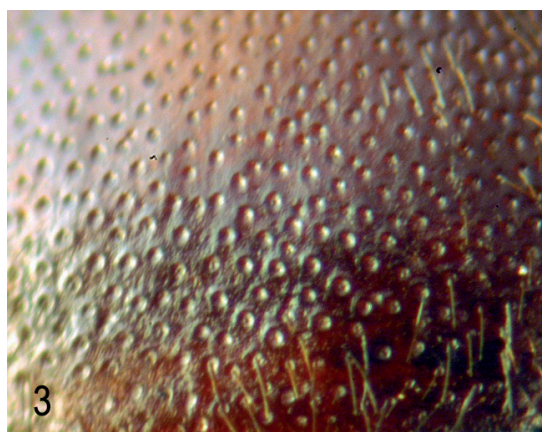

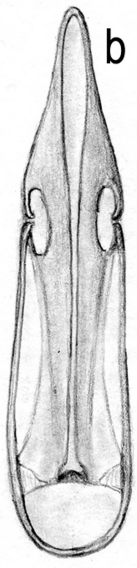

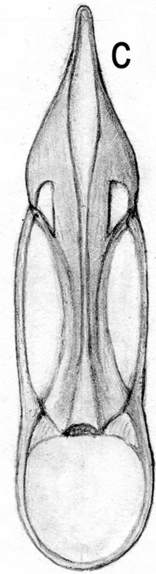

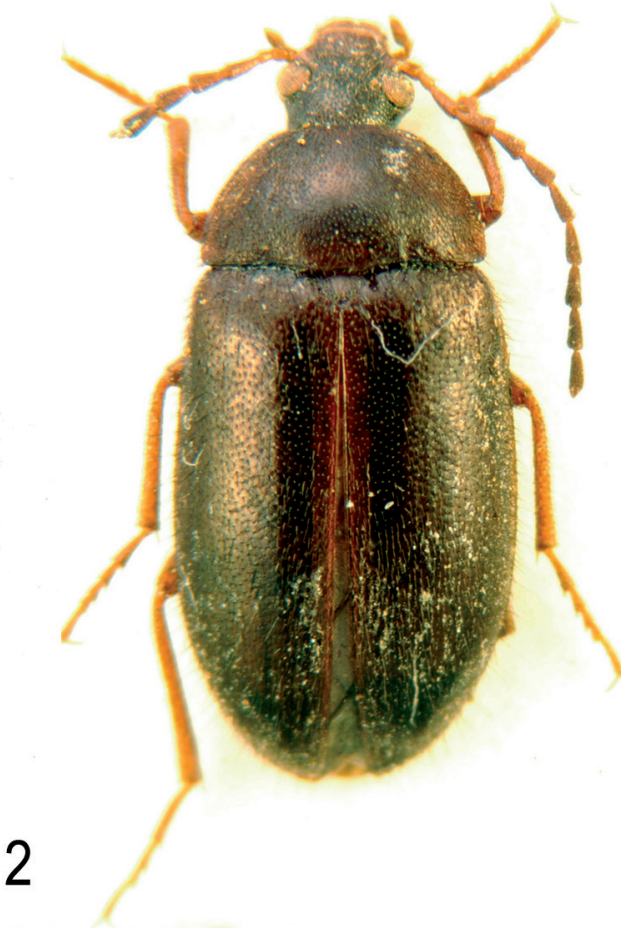

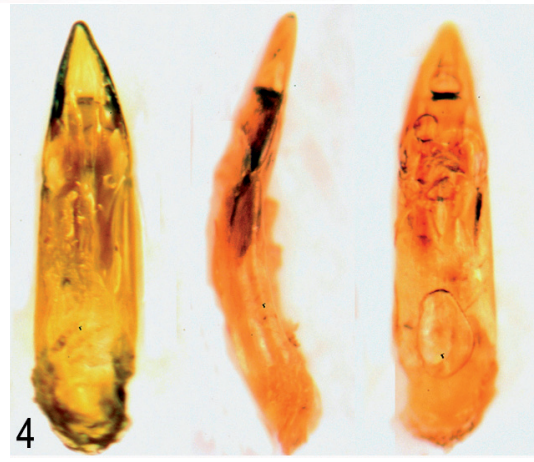

Figs. 2-5.- 2) Habitus dorsal, 3) punteado del pronoto y 4) genitalia masculina en vista ventral (izquierda), lateral (centro) y dorsal (derecha) del Holotypus de Prionychus alfonsoblancoi n. sp. 5) Imágenes comparativas, en vista ventral, de las genitalias de Prionychus alfonsoblancoi n. sp. (a), P. anthracinus (b) y P. lugens (c).

Figs. 2-5.- 2) Dorsal view, 3) pronotal punctuation and 4) male genitalia in ventral view (left), lateral view (center) and dorsal view (right) of the holotype of Prionychus alfonsoblancoi n. sp. 5) Comparative images, in ventral view, of the genitalias of Prionychus alfonsoblancoi n. sp. (a); $P$. anthracinus (b) and $P$. lugens (c). 
Escudete: Triangular, plano, con punteado neto a base de puntos relativamente pequeños que dejan libre una pequeña banda central. Dichos puntos portan sedas como las de los élitros. Tegumento entre los puntos con una microescultura semejante a la de los élitros.

Élitros: Ovales, del mismo color castaño oscuro que el resto del cuerpo (longitud: 5,2 mm; anchura: $1,8 \mathrm{~mm}, \mathrm{~L} / \mathrm{A}: 2,89)$. Relativamente cortos; algo más de tres veces la longitud del pronoto (LE/LP: 3,25). No se aprecian realmente estrías; si bien el alineamiento de los puntos induce a sugerirlas; no obstante a nivel del declive y ápex élitral sí pueden identificarse la dos interestrías yuxtasuturales y muy levemente las dos siguientes. Punteado simple, moderadamente denso, pero menos fuerte que el del pronoto; los puntos separados por tres veces su diámetro. Al igual que el pronoto, el tegumento entre los puntos muestra una microescultura neta que disminuye su brillo. La pilosidad es doble; por un lado sedas de color castaño oscuro y tumbadas de las que emergen otras mucho más escasas, largas, negras y erguidas, situadas sobre todo a los lados y en la mitad anterior de las epipleuras. Éstas últimas bien desarrolladas a lo largo de toda la longitud de los élitros.

Patas (al ejemplar le falta la metatibia y metatarso derechos): Ligeramente más claras que el resto del cuerpo con sedas abundantes de longitud moderada. Tarsos: Protarso: 1,20 mm (Artejos: 0,33; 0,17; 0,$15 ; 0,12 ; 0,43$ ). Mesotarso: $1,45 \mathrm{~mm}$ (Artejos: $0,45 ; 0,22 ; 0,17 ; 0,15 ; 0,45)$. Metatarso: $1,60 \mathrm{~mm}$ (Artejos: 0,$67 ; 0,27 ; 0,20 ; 0,47)$. Penúltimo artejo de cada tarso provistos de lóbulo membranoso. Uñas de los protarsos con 5 dientes.

Región ventral del cuerpo: Coloración semejante a la del dorso. Prosterno plano provisto de puntos gruesos, poco densos y poco profundos. Esternitos con sedas tumbadas de longitud semejante a las de los élitros.

Edeago (Figs. 4 y 5a): Relativamente robusto (L/A: 3,87), con una relación pieza apical/falobase de 0,48. Pieza apical subtriangular con extremo apuntado pero romo y relación largo/ancho: 1,70.

Etimología: Dedicamos esta especie a Alfonso Blanco, hijo de uno de los autores, que murió prematuramente a la edad de diez años.

Distribución: Beni Sicar, actualmente Beni Chiker, era una kabila cercana a Melilla, extendida a uno y otro lado del monte Gurugú, al norte de Nador, región de Guelaia a $15 \mathrm{~km}$ al sur del Cabo Tres Forcas, en la cuenca del Río de Oro (U.T.M.
UE 90). Pensamos que "Muley Bagdad" debe referirse a la Zauia de Abdelkader El Yilali, situada en el monte Arbós.

El recolector se trata de Rafael Candel Vila, geólogo $\mathrm{y}$, por aquel entonces, Catedrático de Ciencias Naturales del Instituto de Melilla. Llevó a cabo trabajos en el Instituto de Rabat y recolectó gran cantidad de ejemplares de insectos, muchos de ellos se encuentran actualmente en el Museo Nacional de Ciencias Naturales de Madrid.

\section{Prionychus lugens (Küster, 1850)}

Material estudiado: 1 macho de Esauira (Mogador), VI/1907, M. M. de la Escalera leg. ( $\mathrm{n}^{\circ}$ cat. 29768). 1 macho de Tetuán, sin fecha, M. M. de la Escalera leg. ( $n^{\circ}$ cat. 30805). Depositados en el MNCN.

REDESCRIPCIÓN (para las mediciones se ha utilizado el ejemplar no 29768 del MNCN): Morfología general oval. De coloración castaño oscuro, con las patas, antenas y palpos ligeramente más claros (Fig. 6). Longitud total desde el borde del clípeo al ápex elitral: 7,7 mm. Anchura máxima en la unión del tercio medio con el tercio posterior de los élitros: 3,6 mm (L/A: 2,14).

Cabeza: Longitud (visible): 1,0 mm. Anchura máxima (a nivel de los ojos): 1,4 mm. De la misma coloración general que el cuerpo, algo más clara en el área del clípeo. Clípeo deprimido con respecto a la frente, lo cual dibuja un fuerte declive clípeofrontal. Ojos moderadamente salientes con una notable distancia interocular: $0,6 \mathrm{~mm}$. lo cual ofrece un índice ocular de 42,86. Punteado de la cabeza fuerte y denso, salvo el clípeo; los puntos de un diámetro mayor que la distancia que los separa, algo mayores que los del pronoto. Tegumento visible entre los puntos liso y brillante. Pilosidad relativamente corta y erguida.

Antenas: Relativamente cortas, con una longitud total de 3,57 mm, no alcanzando la unión del tercio basal de los élitros con el tercio medio (LT/LA: 2,16). Antenómeros muy semejantes entre si; relativamente anchos con respecto a su longitud; fuertemente punteados y provistos de abundantes sedas. Sus longitudes desde la base al ápex (entre paréntesis relación L/A) son las siguientes: 0,36 $(2,05) ; 0,16(1,06) ; 0,31(2,06) ; 0,36(2,06) ; 0,34$ $(1,94) ; 0,31(1,75) ; 0,31(1.82) ; 0,36(2,05) ; 0,36$ $(2,05) ; 0,34(1,79) ; 0,36(2,05)$.

Palpos maxilares: Del mismo color que las antenas, patas y clípeo, con setas relativamente densas, 

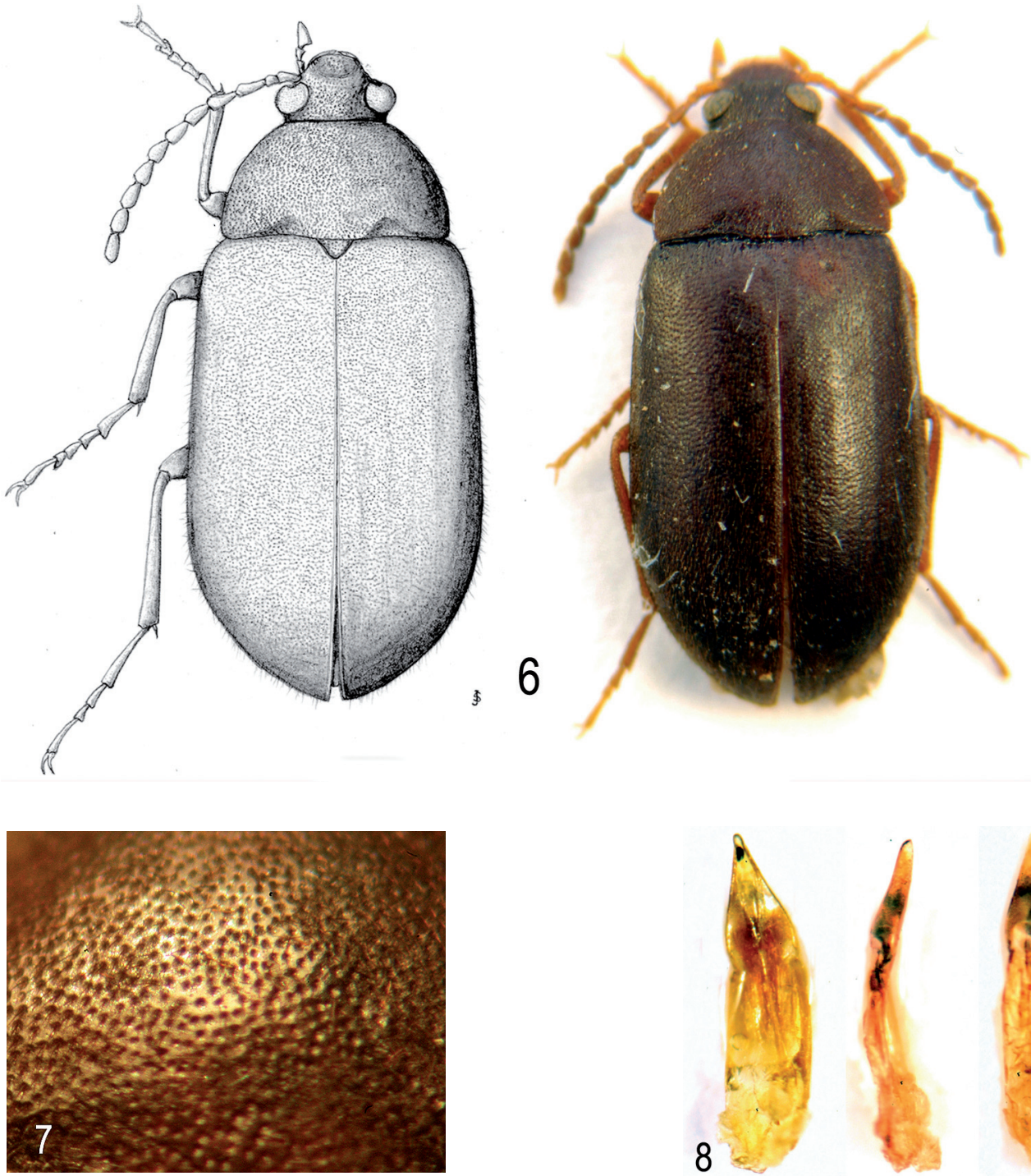

Figs. 6-8.- Prionychus lugens. 6) Habitus dorsal, 7) punteado del pronoto y 8) genitalia masculina en vista ventral (izquierda), lateral (centro) y dorsal (derecha).

Figs. 6-8.- Prionychus lugens. 6) Dorsal view, 7) pronotal punctuation and 8) Male genitalia in ventral view (left), lateral view (center) and dorsal view (right).

especialmente en el último artejo donde, además, aparecen paralelas y tumbadas contra el tegumento. De ellos, el más corto es el penúltimo, el cual tiene una morfología triangular y es tan largo como ancho; el más largo, el último o apical, también con morfología triangular. Las longitudes de los artejos $2^{\circ}$ a $4^{\circ}$ (entre paréntesis relación L/A) son las siguientes: $0,25(2,22) ; 0,125(1,00) ; 0,35(1,75)$.
Pronoto: Coloración uniforme castaño oscuro. Muy transverso, más del doble de ancho que de largo. Longitud: 1,4 mm. Anchura: 2,9 mm. Índice pronotal: 48,27. Rebordeado salvo en el borde anterior, en una longitud que abarca aproximadamente la distancia interocular. Visto lateralmente es escasamente convexo, tanto en sentido anteroposterior como lateral. Punteado denso y moderadamente 
fuerte, más hacia los lados que en el disco. Diámetro de los puntos más o menos igual que la distancia que los separa. Tegumento entre los puntos brillante, sin microescultura (Fig. 7). Pilosidad relativamente larga; erguida en el disco y más o menos tumbada hacia los lados. Angulos posteriores del pronoto vivos en ángulo discretamente obtuso. El pronoto muestra sendas leves depresiones en la región basal paramedial.

Escudete: Triangular, plano, con un punteado neto y uniforme a base de puntos relativamente pequeños, semejantes a los de los élitros.

Élitros: Subparalelos, del mismo color general que el resto del cuerpo. (longitud: 5,6 mm; anchura: $1,9 \mathrm{~mm}$; L/A: 2,95). Cuatro veces más largos que la longitud del pronoto (LE/LP: 4). No existen trazas de estrías salvo en el declive y área apical, donde pueden identificarse las dos primeras y tenuemente la tercera, dibujando sendas interestrías levemente convexas, casi planas. Punteado relativamente denso y uniforme, a base de puntos algo más pequeños que los del pronoto. Entre los puntos el tegumento aparece brillante sin trazas de microescultura. Pilosidad simple, de color castaño, a base de sedas relativamente cortas y tumbadas, sin ninguna erguida.

Patas: Ligeramente más claras que el resto del cuerpo con sedas abundantes de longitud moderada. Protarso: 1,20 mm (Artejos: 0,33; 0,17; 0,15; 0,12; 0,43). Mesotarso: 1,45 mm (Artejos: 0,45; $0,22 ; 0,17 ; 0,15 ; 0,45)$. Metatarso: $1,60 \mathrm{~mm}$ (Artejos: 0,$67 ; 0,27 ; 0,20 ; 0,47$ ). Penúltimo artejo de cada tarso provisto de lóbulo membranoso. Uñas de los protarsos con 5 dientes.

Región ventral del cuerpo: Coloración semejante a la del dorso, aunque algo más clara. Prosterno plano, con puntos gruesos pero dispersos y poco profundos y con frecuencia no completos, semejando más bien depresiones o vermiculaciones, sobre todo cerca del borde anterior.

Edeago (Figs. 8 y 5c): Relativamente ancho en relación a su longitud (L/A: 3,55). Relación entre longitud de la pieza apical en relación con la falobase de 0,60. La pieza apical es característicamente ancha, lanceolada, subcordiforme, sobre todo en visión ventral; bastante aplanada en visión lateral y con una relación largo/ancho de 1,71.

\section{Prionychus anthracinus (Mulsant, 1856)}

Material estudiado: 1 ej. de Tagsut Sehnaia Rif (Xauen), sin fecha, I. Bolívar leg. ( $\mathrm{n}^{\circ}$ cat. 29773); 1 ej. de Zoco Telata, Ketama, Rif (Alhucemas), VI/1932 (n cat. 29769); 5 ejs. de
Zoco Telata, Ketama, Rif (Alhucemas), sin fechas ni captor ( ${ }^{\circ}$ cat. 29770, 29771, 30806, 30807, 30808); 1 ej. de Tizitaka Beni Seddat, Rif (Alhucemas), VI/1932 I. Bolivar leg. ( $\mathrm{n}^{\circ}$ cat. 30809); 1 ej. Tánger-Tetuán, sin fecha, M. M. de la Escalera leg. $\left(n^{\circ}\right.$ cat. 29772$)$ y 1 ej. de Xauen, sin fecha ni captor $\left(n^{\circ}\right.$ cat. 29774). Depositados en el MNCN.

REDESCRIPCIÓN (Para las mediciones se ha utilizado el ejemplar no 29771 del MNCN): Morfología general alargada. Coloración general castaño oscuro con el pronoto y la cabeza aún más oscuros que los élitros. Patas, palpos y antenas algo más claros (Fig. 9). Longitud total desde el borde del clípeo al ápex elitral: $8,1 \mathrm{~mm}$. Anchura máxima en la unión del tercio medio con el tercio posterior de los élitros: 3,4 mm (L/A: 2,38).

Cabeza: Longitud (visible): $1,15 \mathrm{~mm}$. Anchura máxima (a nivel de los ojos): 1,55 mm. Ojos salientes con una distancia interocular de $0,65 \mathrm{~mm}$. lo cual ofrece un Índice ocular de 41,93. Coloración castaño muy oscuro. Clípeo deprimido con respecto a la frente. Punteado fuerte y denso, algo menos fuerte en el clípeo. Los puntos de diámetro mucho mayor que la distancia que los separa, de tal forma que apenas queda tegumento entre ellos. Pilosidad de la misma coloración que el fondo y tumbada.

Antenas: Longitud total: 4,95 mm. Relativamente largas y estilizadas, sobrepasando claramente la unión del tercio medio con el tercio basal de los élitros. Antenómeros fuertemente punteados y provistos de abundantes sedas, de longitudes casi idénticas del cuarto al décimo. Sus longitudes desde la base al ápex (entre paréntesis relación L/A) son las siguientes: $0,30(1,71) ; 0,17(1,16) ; 0,35(2,00) ; 0,50(2,22)$; $0,47(2,23) ; 0,50(2,35) ; 0,50(2,22) ; 0,52(2,33)$; $0,50(2,50) ; 0,52(2,62) ; 0,60(3,42)$.

Palpos maxilares: Del mismo color que las antenas, patas y clípeo, con setas relativamente densas, especialmente en el último artejo donde, además, aparecen paralelas y tumbadas contra el tegumento. De ellos, el más corto es el penúltimo, el cual tiene una morfología triangular; el más largo el último o apical, también con morfología triangular. Las longitudes de los artejos $2^{\circ}$ a $4^{\circ}$ (entre paréntesis relación L/A) son las siguientes: $0,25(2,00) ; 0,15$ $(1,2) ; 0,40(1,77)$.

Pronoto: Coloración castaño muy oscuro. Transverso, menos del doble de ancho que de largo. Longitud: 1,4 mm. Anchura: 2,6 mm. Índice pronotal: 53,84. Rebordeado salvo en el borde anterior, en una longitud que abarca aproximadamente la distancia interocular. Visto lateralmente es poco convexo, tanto en sentido anteroposterior como 

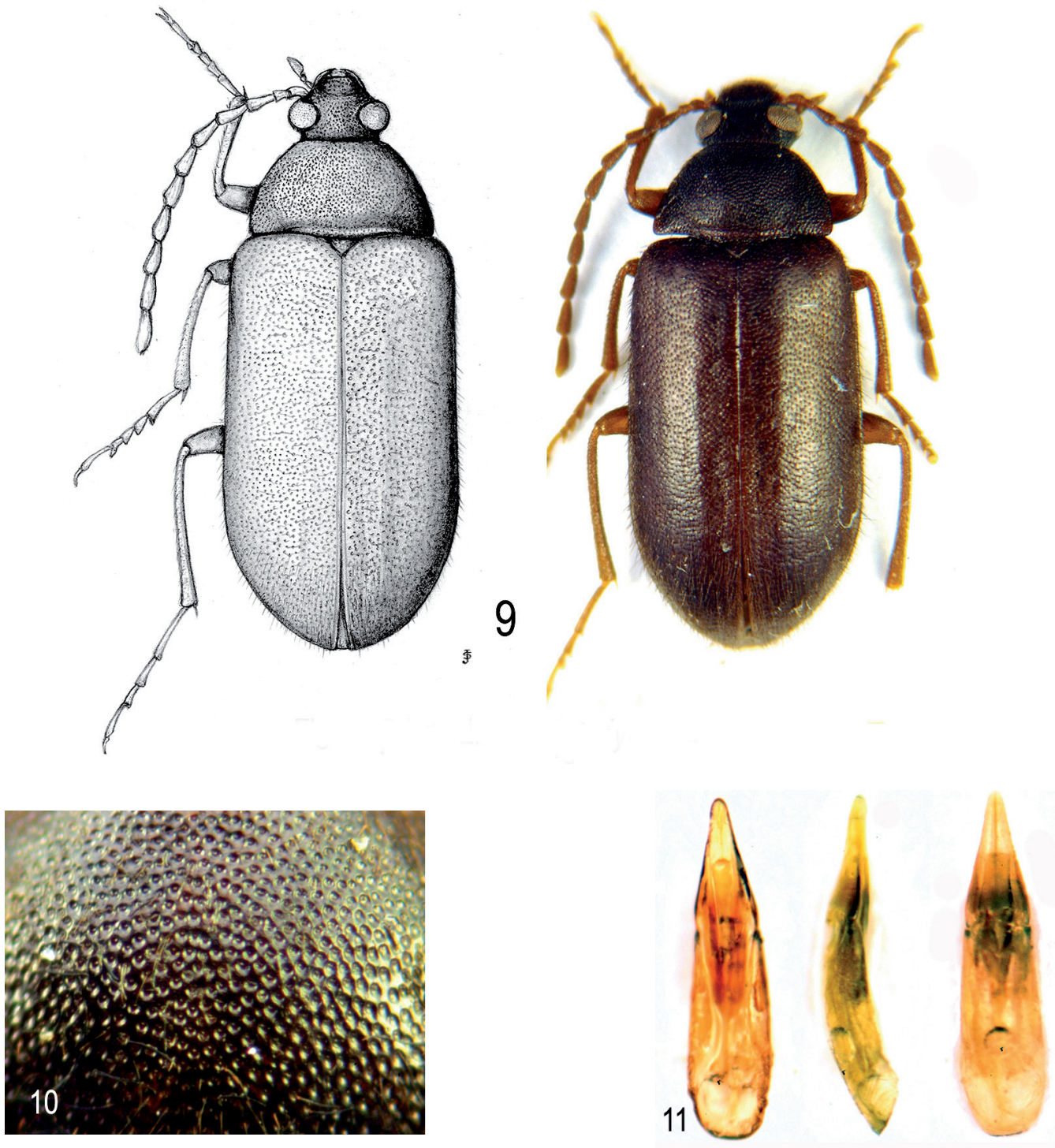

Figs. 9-11.- Prionychus anthracinus. 9) Habitus dorsal, 10) punteado del pronoto y 11) genitalia masculina en vista ventral (izquierda), lateral (centro) y dorsal (derecha).

Figs. 9-11.- Prionychus anthracinus. 9) Dorsal view, 10) pronotal punctuation and 11) male genitalia in ventral view (left), lateral view (center) and dorsal view (right).

lateral. Ángulos anteriores muy redondeados y poco marcados; los posteriores por el contrario aparecen muy marcados y en ángulo recto. Punteado fuerte, denso y uniforme. Los puntos aparecen umbilicados y de diámetro mayor que la distancia que los separa. El tegumento entre ellos es liso y brillante, sin trazas de microreticulación (Fig. 10). Paralela al borde posterior, a poca distancia del mismo y en la zona media, se aprecia una depresión lineal que abarca aproximadamente los $2 / 3$ centrales, desde aproximadamente la mitad de la base elitral a la otra. Pilosidad relativamente larga, de color castaño, algo más claro que el tegumento y tumbada hacia atrás.

Escudete: Triangular con puntos semejantes a los de los élitros, aunque más superficiales. 
Élitros: De color castaño oscuro, menos oscuro que el pronoto y la cabeza. Prácticamente paralelos, apenas más anchos en el tercio basal que en el distal (longitud: 5,6 mm, anchura máxima: $1,7 \mathrm{~mm}, \mathrm{~L} / \mathrm{A}$ : $3,29)$. Cuatro veces más largos que la longitud del pronoto (LE/LP: 4). Sin trazas de estrías, salvo ligeramente las dos primeras en el declive apical. Las dos bandas elitrales paralelas yuxtasuturales, al estar en un mismo plano con respecto al resto de los élitros que forman declive, dan la falsa impresión de estrías, impresión que desaparece con luz cenital. Punteado simple, moderadamente denso, menos fuerte y denso que el del pronoto. Los puntos mucho más pequeños que la distancia que los separa. Tegumento brillante, aunque levemente microreticulado. Pilosidad doble, castaño oscura, con pelos tumbados y erguidos; estos últimos más frecuentes a los lados y en el declive.

Patas: Protarso: 1,37 mm (Artejos: 0,40; 0,18; $0,15 ; 0,12 ; 0,52)$. Mesotarso: $1,64 \mathrm{~mm}$ (Artejos: $0,55 ; 0,27 ; 0,17 ; 0,15 ; 0,50)$. Metatarso: $1,90 \mathrm{~mm}$ (Artejos: 0,$85 ; 0,35 ; 0,20 ; 0,50$ ). Penúltimo artejo de cada tarso provistos de lóbulo membranoso. Uñas de los protarsos con 5 dientes.

Región ventral del cuerpo: Coloración uniformemente castaño oscuro, semejante a la del dorso. Prosterno plano con un punteado denso y fuerte. El diámetro de los puntos supera a la distancia entre ellos.

Edeago (Fig. 11): Relativamente estilizado (L/A: 4,06), con una relación entre la pieza apical y basal de 0,66. La pieza apical es regularmente acuminada pero con el ápex redondeado; relativamente grande con una relación entre L/A de 2,1.

\section{Discusión}

Desde la monumental obra de Seidlitz (1896) la morfología del prosterno se revela como el principal carácter para separar las especies del genero Prionychus. Atendiendo a este carácter, se han separado tradicionalmente en dos grandes grupos: aquellas especies en las que el prosterno, por delante de las procoxas, aparece en declive plano o algo convexo y otras en las que aparece horizontal y cóncavo. En este sentido Prionychus alfonsoblancoi se separa en primer lugar claramente del grupo de especies que tienen el prosterno cóncavo y el borde anterior del pronoto claramente rebordeado; esto es, de Prionychus fairmairii y Prionychus melanarius.
Además estas dos especies son negras y más grandes entre otros muchos caracteres. Importancia taxonómica tiene también la apófisis prosternal según supere posteriormente el nivel de las procoxas o no. En este sentido, Prionychus alfonsoblancoi se separa fácilmente de Prionychus anthracinus (Mulsant, 1856), dado que en esta especie la apófisis prosternal supera claramente el nivel de las procoxas. Además esta especie ofrece otros detalles que la hacen inconfundible; entre ellos la depresion lineal pronotal posterior (anteescutelar), el fuerte punteado del pronoto, la longitud de las antenas, los élitros subparalelos y proporcionalmente más largos, lo cual le confiere un aspecto alargado.

De gran importancia taxonómica es también la estriación elitral, por ello Prionychus alfonsoblancoi también es claramente distinguible de Prionychus ater, dado que, entre otros muchos detalles, la especie de Fabricius tiene los élitros fuertemente estriados; además es negra y mucho más grande.

La especie más semejante sin duda es Prionychus lugens, de la que puede separarse fácilmente en primer lugar por las antenas, que en $P$. alfonsoblancoi son relativamente largas (alcanzan el tercio medio de los élitros) y finas, mientras que en $P$. lugens son relativamente cortas (no alcanzan ni siquiera el cuarto basal de los élitros) y de artejos anchos con formas y proporciones diferentes, especialmente claras en el último artejo. Por otra parte el punteado del pronoto es completamente diferente. También es muy llamativa la diferencia entre las piezas apicales del edeago: casi subcordiforme en $P$. lugens y regularmente acuminada en $P$. alfonsoblancoi (Fig. 5).

De las especies más alejadas geográficamente -asiáticas en su mayoría- y de las que no hemos podido estudiar ejemplares, se diferencia por los siguientes caracteres extraídos de sus descripciones originales (Fairmaire, 1892; Pic, 1905, 1909; Seidlitz, 1896). De P. striatipennis, por ser ésta negra y brillante, con punteado elitral mucho más fuerte que el pronotal y el tercer artejo antenar la mitad de largo que el segundo. P. asiaticus es una especie también negra y glabra, élitros con estrías distintas, ojos muy próximos y las antenas un poco de perfil en sierra. De $P$. nitidissimus, entre otros detalles, por ser negro brillante pero con las patas rojizas. $P$. delagrangei es de color negro y brillante con antenas muy largas. Por último de $P$. cisteloides por tener los penúltimos tarsómeros con lóbulos indistintos y élitros subestriados estando las estrías indicadas por puntos. 


\section{Clave dicotómica de las especies ibéricas y nor- teafricanas de Prionychus}

1. Borde anterior del pronoto no rebordeado en el medio. Prosterno, por delante de las procoxas, en declive plano o algo convexo

1. Borde anterior del pronoto fina pero claramente rebordeado en el medio. Prosterno, por delante de las procoxas, muy corto, horizontal y cóncavo

5

2. Prosterno fuerte, gruesa y densamente punteado. Pronoto con clara depresion transversal anteescutelar y con punteado grueso y muy denso. Castaño muy oscuro. 7,5-9,5 mm. Argelia, Marruecos y Túnez

$P$. anthracinus

2'. Prosterno con punteado esparcido más o menos irregular. Pronoto sin depresión transversal anteescutelar y con punteado más fino y más o menos denso

3. Élitros claramente estriados en toda su superficie. Negro mate. Grande: 12-24 mm. Europa ......... P. ater

3'. Élitros casi lisos, solo con trazas de la primera y segunda estrías yuxtasuturales y tercera y cuarta en el declive y ápex. Castaño más o menos oscuro. Pequeño: 7,5-10 $\mathrm{mm}$

4. Antenas cortas, no alcanzando el tercio medio de la longitud elitral. Punteado del pronoto en el disco denso y fuerte, diámetro de los puntos más o menos igual que la distancia que los separa. Tegumento entre los puntos brillante, sin microescultura. Pilosidad elitral simple y tumbada. 7,5-10 mm. Córcega, Sicilia, Marruecos y Argelia $P$. lugens

4'. Antenas largas, alcanzando sobradamente el tercio medio de la longitud elitral. Punteado del pronoto en el disco menos denso, con diámetro de los puntos menor que la distancia que los separa. Tegumento entre los puntos con microescultura. Pilosidad elitral doble. 7,6 $\mathrm{mm}$. Marruecos

P. alfonsoblancoi

5. Ángulos posteriores del pronoto aproximadamente rectangulares. Estrías elitrales indistintas e interestrías planas. Élitros por lo general mates. Negro. 10-18 mm. Europa sudoccidental, Marruecos ............. P. fairmairii

5' Ángulos posteriores del pronoto distintamente redondeados. Estrías elitrales distintas e interestrías claramente convexas. Élitros por lo general brillantes. Negro. $10 \mathrm{~mm}$. Europa P. melanarius*

* Vive en Francia y gran parte de Europa, su presencia en la península ibérica precisa confirmación.

\section{Agradecimientos}

Debemos dar las gracias a todos los que de una forma $\mathrm{u}$ otra están colaborando para actualizar la corología de los Alleculinae ibéricos. En este sentido debemos mencionar especialmente la colaboración prestada por D. Pablo Bahillo (Baracaldo) y D. José I. López-Colón (Madrid), con quienes venimos trabajando desde hace años en distintas familias de coleópteros. A D. José Luis Lencina (Murcia), D. Diego Gallego (Murcia), D. Carmelo Andújar (Hellín), D. Eudaldo
González (Valencia), D. José Luis Torres (La Línea) y D. Pedro Coello (San Fernando), quienes nos prestaron para su estudio los ejemplares del género Prionychus contenidos en sus colecciones. D. Vladimir Novák (República Checa), D. Fabien Soldati (Troyes, Francia) y D. Rafael Yus (Málaga) nos prestaron material europeo de $P$. melanarius, $P$. fairmairii y $P$. ater.

Muy especialmente debemos dar las gracias al Museo Nacional de Ciencias Naturales de Madrid, el cual a través de Mercedes Paris, puso a nuestra disposición los ejemplares de Prionychus contenidos en sus colecciones entomológicas. Debemos también dar las gracias a Manuel Sánchez Ruiz por su apoyo constante y por ayudarnos a localizar bibliografía difícil de encontrar. A todos ellos expresamos nuestro más sincero agradecimiento.

\section{Referencias}

Campbell, J. M., 1971. A revision of the Alleculidae (Coleoptera) of the West Indies. Memoirs of the Entomological Society of Canada, 81: 3-140.

Campbell, J. M. \& Marshall, J. D., 1964. The ocular index and its application to the taxonomy of the Alleculidae (Coleoptera). The Coleopterist's Bulletin, 18: 42.

Fairmaire, L. M. H., 1892. Descriptions de coléoptères des environs d'Akbès (Syrie). Annales de la Societé Entomologique du Belgique, 36: 144-159.

Küster, H. C., 1850. Die Käfer Europa's. Nach der Natur Beschrieben. Heft 20. Bauer \& Raspe. Nürnberg. [4] +100 sheets, 2 pls.

Mulsant, E., 1856. Notes relatives à quelques insectes coleóptères de la tribu des Pectinipèdes. Opuscules Entomologiques, 7: 17-59.

Novák, V., 2006. Hymenophorus evae sp. nov. and H. gerdae sp. nov. (Coleoptera: Tenebrionidae: Alleculinae) from Irán. Acta Societatis Zoologicae Bohemicae, 69: 317-325

Novák, V., 2007a. A new genus and three new species of Alleculinae Coleoptera: Tenebrionidae) from Socotra Island, Yemen. Fauna of Arabia, 23: 319-334.

Novák, V., 2007b. New species of the genus Hymenalia Mulsant, 1856 (Coleoptera: Tenebrionidae: Alleculinae) from Palaearctic region. Studies and reports of District Museum Prague-East, Taxonomical series, 3(1-2): 149-170.

Novák, V., 2008a. Order Coleoptera, family Tenebrionidae. In: A. van Arten (ed.). Arthropod fauna of the United Arab Emirates, Vol. 1. Dar Al Ummah Printing. Abu Dhabi: 257-263.

Novák, V., 2008b. Mycetocharina (Alleculopsis) bahukalatensis sp. nov. (Coleoptera: Tenebrionidae: Alleculinae) from Iran. Acta Entomologica Musei Nationalis Pragae, 48(1): 73-78.

Novák, V. \& Petterson, R., 2008. Subfamily Alleculinae. In: I. Löbl \& A. Smetana (eds.). Catalogue of Palaearctic 
Coleoptera. Vol. 5 Tenebrionoidea. Apollo Books. Stenstrup: 319-338.

Pic, M., 1905. Coléoptères asiatiques nouveaux. Bulletin de la Société Entomologique de France, 1904: 287289.

Pic, M., 1909. Descriptions ou diagnoses et notes diverses (Suite). L'Echange, Revue Linnéenne, 25: 113115.

Sáez, J. A., Blanco, J. M., Bahillo, P. \& López-Colón, J. I., 2010. Aportación a la corología del género Prionychus Solier, 1835 (Coleoptera: Tenebrionidae, Alleculinae) en España y Marruecos. Boletín de la Sociedad entomológica Aragonesa, 47: 22-234.

Seidlitz, G. C. M. von., 1896. Alleculidae. In: Naturgeschichte der Insecten Deutschlands. Begonnen von Dr. W. F. Erichson, fortgesetz von Prof. Dr. H. Schaum, Dr. G. Kraatz, H. v. Kiesenwetter, Julius Weise, Edm.
Reitter und Dr. G. Seidlitz. Erste Abtheilung Coleoptera. Fünfer Band. Zweite Hälfte. Lieferungen 1-3. Nicolaische Verlags-Buchhand-lung. Berlin: 1305.

Aceptado / Accepted, 22-09-2011 Publicado impreso / Published in print, 30-12-2011 